\title{
The Needs of Group Counseling for Ethnic Minority College Students from the Perspective of Social Psychological Service
}

\author{
Yuxi Liu, Shicheng Li \\ School of Education and Psychology, Southwest University for Nationalities, Chengdu, China \\ Email: 1093928878@qq.com
}

How to cite this paper: Liu, Y.X. and Li, S.C. (2021) The Needs of Group Counseling for Ethnic Minority College Students from the Perspective of Social Psychological Service. Open Access Library Journal, 8: e7224. https://doi.org/10.4236/oalib.1107224

Received: February 5, 2021

Accepted: March 13, 2021

Published: March 16, 2021

Copyright () 2021 by author(s) and Open Access Library Inc.

This work is licensed under the Creative Commons Attribution International License (CC BY 4.0).

http://creativecommons.org/licenses/by/4.0/

\begin{abstract}
With the development of social economy and the improvement of learning quality and quality education requirements, the social attention to the psychological health of college students and social adaptability needs is also more and more high. The research shows that, due to the influence of various factors such as region, economy and culture, the social service needs of minority and Han university students will also be different at different levels. As the hope and future of all ethnic groups, minority college students are the backbone of inheriting, developing and innovating the traditional culture of ethnic groups. It is an important aspect of the development of ethnic education to pay attention to the psychological status of such a special group. At present, there are a lot of researches on the psychological health service needs of college students at home and abroad, but the researches on the psychological health service needs of minority college students are less, and mainly focus on the application. Therefore, the research on the needs of minority college students for group counseling and group counseling is of great theoretical significance.
\end{abstract}

\section{Subject Areas}

Psychology

\section{Keywords}

Minority College Students, Group Counseling, Demand

\section{1. 引言}

团体咨询是在团体情境下进行的一种心理咨询形式, 在帮助那些有着共同发 
展课题和相似心理困扰的人时, 团体心理咨询较个别咨询而言更加经济有效 (邵瑾, 刹富珉, 2015 [1]; 王茹婧等, 2017 [2])。根据少数民族大学生的心理 发展状态, 合理利用团体心理辅导, 有针对性地对学生进行及时、有效的心 理指导, 提高他们适应社会的能力, 培养他们良好的心理素质, 使其身心得 到良好、协调发展, 已经成为各个民族院校进行心理健康教育的重要任务。

需求是指有机体内部的某种缺乏或不平衡的状态, 心理健康服务需求则 是指个体当前的心理健康水平和想要达到的心理健康水平之间存在的差距所 引起的缺乏或不平衡状态(方芳, 2019) [3]。少数民族大学生对团体咨询的需 求研究现状可以拆分为两个方面, 一是对于当代大学生心理健康服务需求的 研究现状; 二是团体咨询与团体辅导在少数民族大学生中的实践应用现状。 同时, 在对当代大学生心理健康服务需求的研究现状中, 又可以细分为三部 分, 分别为: 当代中国大学生心理健康服务需求的研究; 不同方式的团体辅 导和团体咨询对大学生的影响; 团体辅导和团体咨询对大学生不同阶段心理 健康服务需求的影响。

\section{2. 当代中国大学生的心理服务需求}

人格发展是心理成长的重要课题, 自我的实现是人格发展过程中的核心 问题。在埃里克森的自我发展理论中, “自我同一性” 这一概念是其中重要 的组成部分, 获得同一感是青春期发展的核心任务。青少年从对父母的依赖 关系中逐渐脱离出来, 形成独立的个体, 他们会对周围世界产生新的观察和 新的思考, 他们会经常考虑自己是怎样一个人, 会从他人对自己的态度中, 也会从自己扮演的各种社会角色中, 逐渐认清自己, 体验到自我内在的延续 感, 确立自己的身份。成功获得同一性, 会帮助青少年顺利进入成年期, 否 则就会产生同一性混乱, 无法获得自我身份的确立, 难以和他人建立亲密关 系。

但其中还有另外一种情况, 即 “延期偿付 1 ”, 青少年可能会推迟这一任 务, 延迟做出个人生活或职业的选择和承诺。这一情况在中国社会的复杂背 景下其实较为普遍, 青少年在进入大学之前, 都与父母保持较为紧密的关系, 学习和生活的主题被他人安排。但当其成为一名大学生之后, 这一任务不可 避免地会到来。而另一方面, 大学生作为社会中受教育水平较高的群体, 也 承担着社会更多的期待和压力。这意味着, 大学生不仅需要实现自我确证的 任务, 还会面对更多的社会责任, 他们的心理健康状况值得受到更多的关注。

\section{1. 大学生心理健康服务需求}

总体上来说, 我国大学生的心理健康状况不容乐观, 在校大学生出现心 理问题的比例在三成左右, 而存在较严重心理障碍的大学生约占一成(黄希 庭, 郑涌, 2009) [4]。黄希庭等(2011) [5]对 35 所高校 5058 名的大学生心理 健康服务需求进行的调查, 发现大学生对心理健康服务的需求处于 “有点希 埃里克森用 “延期偿付” 来描述青少年在面临各种选择的思想斗争过程中，会延迟做出个人生 活或职业的选择和承诺。埃里克森认为, 在一个复杂社会, 在这个 “延期偿付” 的阶段, 青少 年势必会经历自我同一性危机, 但对大多数人来说, 自我同一性的达成是一个逐渐缓慢的探索 过程, 而不是外在的急剧变化。延期选择很正常, 而且是健康有益的。 
望” 和 “希望”之间，总体偏向希望得到有效的心理健康服务。

在服务内容上，对人际关系(91.98\%)、择业和职业发展 $(90.4 \%) 、$ 学业问 题(89.9\%)的需求最高, 其次为自己的身心管理 $(88.2 \%) 、$ 生计与生活 $(84.4 \%)$ 以及婚恋和两性心理(69.6\%), 反映出当代大学生的心理健康服务需要聚焦在 对良好人际关系追求、事业发展和学习进步等发展性需要上。

在服务方式和途径上，“科普宣传、健康教育、向同学朋友咨询” 是当 前大学生首选的获得心理健康服务的方式和途径, 而 “与医生、精神科专家 面谈和电话咨询” 被排在最末三位。这些途径体现出中国大学生在心理健康 服务需要表达上的社会取向的特征。

另外，在服务态度上， $84.3 \%$ 的大学生希望有固定的心理健康服务机构提 供服务, $83.2 \%$ 的大学生认为自己有必要了解心理健康知识; $74 \%$ 的大学生认 为有必要和心理健康服务人员讨论自己和家人的心理健康问题。反映出当前

大学生群体有较强的心理服务需要和较高的心理健康服务意愿。

\section{2. 不同方式的团体辅导和团体咨询对大学生的影响}

当前对团体辅导和团体咨询对大学生影响的研究, 很大一部分集中于不 同方式的团体辅导和团体咨询对于大学生的影响研究。如表达性艺术治疗、 叙事疗法、积极心理学取向、焦点解决取向和接纳承诺疗法等对于大学生心 理健康的应用。不同方式团体辅导的研究结果都表明团体辅导和团体治疗对 于大学生心理健康水平的提高有显著性的结果。

在森田疗法取向下团体辅导改善大学生面试焦虑的对照研究中, 采用心 理问卷测量、患者自我评价以及专家评估相结合的方法, 以森田疗法为基础 的团体辅导应用于面试焦虑障碍的治疗。通过实验组、对照组和榜样组之间 的对比测试，在后侧、追踪测试中，实验组中的 SADS 总分和 2 个因子分均 低于对照组; 前测中，实验组 SADS 总分和 2 个因子分均高于榜样组。且实 验组自我量化报告后测得分均高于前测。包括实验组在团体结束后的一周后 填写自我质性报告, 结果也显示了受测者在经过森田疗法, 焦虑情况得到了 有效的改善和提升，总体有效率 90.5\% (倪士光, 伍新春, 张崩, 2010) [6]。

同样, 在团体箱庭疗法干预大学生心理复原力的过程与效果中, 采用干 预组、控制组前后测设计，被试为 19 名低心理复原力大一学生。干预组被试 进行 8 次团体活动, 2 6 次为团体箱庭活动。在其前后测的结果对比中, 我 们也发现: 干预组的《自我复原力量表》和《大学生心理健康问卷(UPI)》分 数变化显著, 控制组不显著; 在箱庭的各个心理复原力评估指标及总分上, 干预组和控制组前测不显著, 后测干预组均显著高于控制组(王丹，张日昇， 2014）[7]。由此可知, 团体箱庭能够有效的干预低心理复原力大学生的心理 复原力水平, 进而改善心理健康水平, 团体箱庭活动中加入心理复原力常见 组成成分自我反思的设计具有一定作用。

而在团体绘画心理辅导在完善大学生自我概念中的作用研究中，其主要 目的是探讨团体绘画心理辅导对帮助大学生完善自我概念的作用。在该研究 中招募 53 名大学生作为团体成员, 进行为期 2 个月, 共 7 个单元的团体绘画 心理辅导。辅导前后采用田纳西自我概念量表对团体成员进行对比测试。其 
结果也有效的显示了团体成员在家庭自我(FA)、自我满意(SA)、道德自我 $(\mathrm{ME}) 、 心$ 理自我(PER)、社会自我(SO)、自我认同(ID)等六个因子有显著提高。 最后的结论也证明了, 团体绘画心理辅导对于帮助大学生完善自我概念是有 效的(雒力静等, 2011) [8]。

\section{3. 团体辅导和团体咨询对大学生不同阶段心理健康服务需求的 影响}

对于不同阶段的大学生来说, 对于心理健康服务的内容需求也会存在不 同的倾向。例如大一阶段的新生, 会更关注于新生适应性和人际交往问题。 而在大二、大三阶段, 会更关注于学习压力问题、情感心理问题等问题。大 四阶段, 主要是为了做好实习和就业准备。对于不同阶段的大学生, 开设不 同类型的团体辅导与咨询, 并对其影响进行研究。如针对大一新生来说, 研 究人际关系小组的效用, 针对大二大三学生, 开设压弹成长小组、两性成长 小组等团体, 对于大四的学生, 研究自我成长小组、职业生涯规划小组等对 大学生的影响。

\section{3. 中国少数民族大学生的心理服务需求}

从客观上来看, 少数民族在地区、经济、政策以及民族文化都存在其特 殊的条件, 这些因素给少数民族大学生带来的心理上的影响, 可能会使得少 数民族大学生的心理健康状况与普通大学生有所不同(杨晓梅, 2011) [9]。总 的来说, 少数民族大学生大多来自经济、文化和教育相对落后的少数民族聚 居地, 在这些特定的生长环境下, 他们形成了勤劳善良、意志坚强等好的品 质, 但另一方面, 作为大学生, 面对一个更具开放性, 竞争也更加激烈的环 境, 他们可能会遇到更多适应性的困难, 心理问题的易感性也可能更高。

\section{1. 中国少数民族大学生的心理健康状况}

由于民族的多样性和分散性, 目前国内对于少数民族大学生心理健康状 况的研究重点各有不同, 往往只偏向于一个民族或地区的研究, 主要的研究 核心是民族文化问题(蔡笑岳, 罗列, 何伯锋, 2012) [10]。此外, 针对于少数 民族大学生这一群体的心理健康状况调查的文献依旧不多, 截止到 2020 年 3 月, 以 CNKI 数据库为检索工具, 以 “心理健康” 、“少数民族大学生” 为 关键词进行检索, 关于少数民族大学生心理健康有 372 条结果, 其中大部分 为教育与思政方面的研究, 心理学上的研究依然较少。

另一方面, 多项研究数据显示, 少数民族大学生心理健康水平总体上较 汉族学生更低。少数民族大学生在进入大学之后, 在文化背景、语言、生活 习惯上面临着更大的差异, 加上部分少数民族学生缺乏良好的自我调节能力, 相比汉族学生, 更容易产生不同程度的心理问题, 主要表现在学业压力、人 际交往、文化适应以及其他方面(吴大鹏, 何萍, 任晓虹, 2020) [11]。根据罗 鸣春(2010) [12]对于中国大学生心理健康服务需求的调查, 发现少数民族大学 生对心理健康服务的需求总体高于汉族学生。根据少数民族大学生心理健康 
调查资料显示, 少数民族大学生的心理素质从总体上看是比较好的, 但存在 的问题也不可忽视。据统计, 少数民族大学生存在不同程度心理问题的占大 学生总数的 $23 \%$, 主要表现在: 环境适应不良、人际交往障碍、自卑、情感 心理问题、学习压力问题五个方面。

\section{2. 团体咨询与团体辅导在少数民族大学生中的实践应用现状}

不同民族拥有其特有的心理健康观念和心理健康维护机制, 而目前国内 针对于少数民族大学生的团体辅导和团体咨询需求的研究还较少, 主要集中 在应用方面, 且从两方面的研究入手。一方面研究不同方式的团体辅导和团 体咨询对于少数民族大学生的应用, 如研究箱庭、心理剧和音乐治疗等技术 在少数民族团体辅导中的应用, 林孟晖(2016) [13]采用团体箱庭疗法对 5 组 40 名新疆少数民族大学生进行干预, 发现团体箱庭能有效改善少数民族大学 生的心理健康水平, 张秀琴(2014) [14]等认为, 在对少数民族大学生开展心理 健康教育时, 在民族认同、人际交往、情绪管理、职业规划等方面, 叙事取 向的团体辅导会更有价值; 另一方面关注团体辅导和团体咨询对于少数民族 大学生不同问题的研究, 包括研究团体辅导对于少数民族大学生适应性、职 业规划和人际交往等方面的影响, 例如范凌云等(2019) [15]通过团体辅导方式 对 82 名少数民族护理实习生职业规划进行干预, 并取得了正向结果, 刘桂芬 等(2007) [16]将团体咨询与个别咨询结合起来对 61 名少数民族大学生进行自 信心训练, 结果发现团体心理咨询可以有效地提高少数民族大学生的自信心。

\section{3. 中国少数民族大学生的心理服务需求展望}

需要意识到的是, 虽然少数民族大学生的心理健康状况显示, 他们需要 更多的心理服务资源, 并不会意味着他们更愿意主动接受心理辅导或心理咨 询服务。研究发现, 汉族大学生与少数民族大学生在心理健康服务的态度和 方式上差异显著, 这表明不同民族特有的心理健康观念和心理健康维护机制 可能影响到其成员对待心理健康服务的态度、求助方式和行为(黄希庭 等, 2011）[5]。少数民族大学生对于服务态度与方式上的需求强度显著高于汉族 学生, 这说明对于少数民族大学生来说, 他们对于获得心理健康服务需求的 方式或途径更为在意, 有必要进一步研究少数民族大学生对于心理健康服务 方式的需求上存在的特点。

另外, 对于不同年级的少数民族大学生来说, 他们对于心理服务的需求 可能也各有不同。根据黄希庭(2011) [5]对中国大学生心理健康服务需求的调 查结果显示, 大学生心理健康服务需要强度呈现 “前高后低” 现象, 即大一、 大二学生的心理健康服务需要显著高于大三、大四学生, 可能是大学新生面 临学习和生活的双重适应, 需要更多的心理社会资源支持, 导致心理健康服 务需要增加, 并在大二阶段集中体现出来。因此, 对于少数民族大学生来说, 不同年级可能也存在不同的心理服务需求, 比如对大一阶段的少数民族学生 来说, 他们可能更需要在新生入学环境适应问题和人际交往问题上得到帮助。 对于大二、大三阶段, 可能更需要处理学习压力问题和情感心理问题。而大 四阶段, 更需要在实习和就业问题上得到帮助(李丹, 尧国靖, 2011) [17]。 


\section{4. 结论与不足之处}

本文主要在对当代中国大学生心理健康服务需求讨论的基础上, 进一步 探讨了在社会心理服务视角下, 少数民族大学生对于团体咨询服务的需求。 总的来说, 目前国内对于少数民族大学生心理健康状况的研究依旧有限, 而 研究数据显示, 少数民族大学生对于心理健康服务存在需求, 并且在需求方式 或需求结构上, 存在其独特的特点。因此, 对于少数民族大学生的心理健康服 务需求进行更直接深入的研究, 从而进行更有针对性的心理服务是必要的。

本文主要是梳理已有的文献中对于少数民族心理健康状况的研究, 发现 目前的研究主要是侧重于应用方面, 例如研究者们更侧重于研究不同的团体 辅导方式对于少数民族大学生心理健康水平的干预效果, 或者团体辅导对于 少数民族大学生不同方面问题的干预情况, 但在实施团体干预之前, 基本没 有对少数民族大学生心理健康服务相关团体主题需求进行调查研究。同样, 这也是本研究的不足之处, 目前研究主要是对已有文献的梳理, 而没有进行 实证性的调查。

其次, 本文只是在概括性的谈论 “少数民族大学生” 这一群体, 并简要的 与汉族大学生心理健康水平与需求进行比较。但少数民族大学生之间也存在差 异, 不同的民族也有其独特的民族语言、文化与信仰, 其心理特点与心理易感 性也会存在不同程度、不同方向的差别; 同时, 即使是同一民族, 由于其成长 环境的差异, 也会为其心理发展带来相应的影响, 例如, 有研究者发现, 内地 藏族学生中, 来自日喀则地区的藏族学生在抑郁、不受欢迎、自伤、思维障碍、 违纪因子上的得分要明显高于拉萨地区的藏族学生(何艳等, 2000) [18]。

因此, 未来对于少数民族大学生团体心理辅导需求的研究应该通过问卷、 访谈或实验等方式, 从实证的角度出发, 考虑到少数民族大学生具体的民族、 年级、居住地、学习经历、学习汉语时间等变量, 了解哪些变量与少数民族 大学生的心理健康水平存在更高的相关, 了解少数民族大学生对于心理健康 服务以及团体辅导的接受度, 从而更好地并且更有针对性的为少数民族大学 生提供心理健康服务。

\section{基金项目}

西南民族大学研究生创新型科研项目(项目编号 CX2020SP56)。

\section{Conflicts of Interest}

The authors declare no conflicts of interest regarding the publication of this paper.

\section{References}

[1] 郡瑾, 㚞富珢. 1996-2013 年国内团体咨询研究的现状与发展趋势 [J]. 中国心理卫 生杂志, 2015, 29(4): 258-263.

[2] 王茹婧, 樊富珢, 李虹, 倪士光. 2001-2016 年国内团体心理咨询效果评估: 现状, 问题与提升策略 [J]. 中国临床心理学杂志, 2017, 25(3): 577-582.

[3] 方芳. 我国心理健康服务需求研究现状和展望[J]. 沈阳大学学报(社会科学版), 2019, 21(3): 324-337. 
[4] 黄希庭, 郑涌. 大学生心理健康教育 $[\mathrm{M}]$. 上海: 华东师范大学出版社, 2009.

[5] 黄希庭, 郑涌, 罗鸣春, 苏丹, 陈本友. 中国大学生心理健康服务需要调查与评 估[J]. 西南大学学报(社会科学版), 2011, 33(3): 1-5.

[6] 倪士光, 伍新春, 张蒋. 森田疗法取向团体辅导改善大学生面试焦虑的对照研究 [J]. 中国心理卫生杂志, 2010, 25(5): 375-379.

[7] 王丹, 张日昇. 团体箱庭疗法干预大学生心理复原力的过程与效果 [J]. 中国临床 心理学杂志, 2014, 22(5): 923-929.

[8] 䧷力静, 李春报, 周爱保, 杨娟, 何随福. 团体绘画心理辅导在完善大学生自我 概念中的作用研究[J]. 中国临床心理学杂志, 2011, 19(4): 558-560.

[9] 杨晓梅. 团体心理咨询对少数民族大学生心理健康教育的实践及探索 [J]. 黑龙江 省社会主义学院学报, 2011, 65(2): 55-56.

[10] 蔡笑岳, 罗列, 何伯锋. 我国西南少数民族心理研究的基本状况 [J]. 心理科学进 展, 2012, 20(8): 1145-1151.

[11] 吴大鹏, 何萍, 任晓虹. 少数民族大学生心理状况现状综述 [J]. 文学教育, 2020, 16(9): 150-151.

[12] 罗鸣春. 中国青少年心理健康服务需求现状研究[D]: [博士学位论文]. 重庆: 西 南大学, 2010.

[13] 林孟晖. 团体箱庭疗法对新疆少数民族学生心理健康水平提升的研究 [J]. 大学教 育, 2016, 5(9): 50-51.

[14] 张秀琴, 何瑾, 焚富枨. 叙事心理治疗及其在少数民族大学生团体辅导中的应用 [J]. 民族教育研究, 2014, 25(5): 17-21.

[15] 范凌云, 刘娜, 薛莲洁, 孙建华. 团体辅导对少数民族护理实习生职业规划影响 [J]. 实用临床护理学电子杂志, 2019, 4(35): 171-179.

[16] 刘桂芬, 王智. 团体咨询结合个别咨询提高少数民族大学生自信水平效果评价 [J]. 中国学校卫生, 2007, 28(12): 1072-1073.

[17] 李丹, 尧国靖. 论团体心理咨询在少数民族大学生心理健康教育中的巧用[J]. 广 西教育学院学报, 2011(6): 139-145.

[18] 何艳, 杜亚松, 孙惠领, 林崇光, 忻仁娥, 唐慧琴, 徐搯园, 刘渏. 藏、汉族的中学 生行为问题的比较研究 [J]. 上海精神医学, 2000, 12(4): 194-197.

\section{Appendix (Abstract and Keywords in Chinese)}

社会心理服务视角下少数民族大学生团体咨询的需求

摘要 随着社会经济的发展以及对学习质量和素质教育要求的提升, 社会对于 大学生的心理健康和社会适应性需求的重视程度也越来越高。研究表明, 因 其地域、经济和文化等多方面因素的影响, 少数民族大学生与汉族大学生的 社会服务需求也会出现不同层次上的差异。少数民族大学生作为各民族的希 望和未来, 是继承、发扬和创新民族传统文化的骨干, 关注这样一个特殊群 体的心理状况, 是民族教育发展的重要方面。目前国内外对于大学生心理健 康服务需求的研究颇多, 但是对于少数民族大学生的心理服务需求的研究较 少, 且主要集中在应用方面。因此, 少数民族大学生对于团体辅导和团体咨 询的需求研究具有十分重要的理论意义。

关键词 少数民族大学生, 团体咨询, 需求 